\title{
Enfoque interpretativo en la corrección de artículos científicos
}

\section{Interpretive approach in the correction of scientific articles}

\author{
Elsy Medina \\ emedina@uc.edu.ve \\ https://orcid.org/0000-0001-9165-2984 \\ Universidad de Carabobo, Valencia, Venezuela.
}

\begin{abstract}
Recibido: 25/08/21
Resumen

Aceptado: 08/11/21

En este trabajo se expone un enfoque interpretativo sobre la escritura en el contexto del artículo científico. Metodológicamente el análisis documental está orientado al aspecto de forma lo que implica mirar a través del lenguaje sobre las posibilidades de elementos básicos y su imbricación con la escritura. Como punto de partida se estudió el corpus lingüístico extraído de diez artículos analizados. Seguido de un apartado basado en las proposiciones ontológicas de Echeverría (2010), las características y tipo oracional, según la UNESCO (1983). Un tercer apartado está constituido por el enfoque interpretativo que ofrece una ruta de lectura para la valoración de la especificidad lingüística dispuesta en los componentes intrínsecos al proceso de corrección de estilo.
\end{abstract}

Palabras clave: Escritura, artículo científico, corrección de estilo.

\section{Abstract}

This work presents an interpretive approach to writing in the context of the scientific article. Methodologically, documentary analysis is oriented to the aspect of shape, which implies looking through language about the possibilities of basic elements and their interweaving with writing. As a starting point, the linguistic corpus extracted from ten refereed articles was analyzed. Followed by a section based on the ontological propositions of Echeverría (2010), the characteristics and type of sentence, according to UNESCO (1983). A third section constituted by the interpretive approach that offers a reading route for the assessment of the linguistic specificity arranged in the intrinsic components of the style correction process.

Keywords: Writing, scientific article, proofreading. 


\section{Introducción}

Este trabajo tiene como objetivo ofrecer una perspectiva interpretativa del lenguaje en el contexto de la escritura de un artículo científico. Por la amplitud y complejidad le subyace un número infinito de características y aspectos propios de esta disciplina. Sin embargo, con el afán de brindar algunas especificidades de acceso lingüístico, el enfoque recae sobre tres apartados que permitirán al investigador construir su propia conciencia lingüística y su respectiva aplicación en la producción escrita.

La comunicación representa un poderoso medio de divulgación del conocimiento en medio de una cultura digital, cuyas redes de conexión han superado la imaginación de cualquier futurólogo. Autores de todo el mundo hacen pública sus investigaciones con argumentos que han sistematizado tras un procesamiento de información; tengamos presente que todo ocurre según el orden de un discurso estructurado para una validación de la comunidad científica.

Consecuente con lo antes expuesto y por representar una importante contribución en consideración del tema en estudio, se destaca la rigurosidad de la escritura, especialmente lo relacionado con el estilo de los artículos científicos señalados por la Unesco 1983, cuyo privilegio es otorgado a la categoría y forma textual; características de estilo de los artículos científicos y la tipología oracional, descritos en lo sucesivo.

Para el presente estudio se empleó la interpretación en el sentido de favorecer la importancia que sobre el lenguaje recae cuando se deja por escrito una publicación como prueba testimonial de un proceso investigativo.

La revisión documental y la sustentación ontoepistémica que fundamenta el interés de esta investigación determinó la posibilidad de sistematizar, en tres apartados, una ruta de lectura que conduce a la valoración de elementos lingüísticos, tales como la metódica, proposiciones ontológicas y el enfoque interpretativo. Se configuran en el momento conclusivo los aspectos intrínsecos de la escritura como orden del discurso y que sin duda se cierne en lo formal, tal como veremos en la importancia atribuida a la corrección de estilo. En su aspecto conceptual, se entiende el valor que tiene la escritura como medio de expresión y su contexto de significación para ordenar las ideas que ocurren en el lenguaje.

\section{Metódica}

En esta investigación de carácter documental se aplicó el análisis, según Berelson (1952); por su consideración descriptiva y sistemática del contenido manifiesto en las comunicaciones y su objetivo de interpretarlas. Es conocido que el interés de este tipo de análisis se debe a la importancia de la información según la unidad de análisis seleccionada y que justamente exige un tipo de interpretación. 
El objetivo metodológico permitió aplicar la hermenéutica para llegar al entendimiento como medio de articulación de la comprensión basado en el aspecto conceptual de Gadamer (2006), "La lectura como un hecho semántico, propio y concluso". En este sentido, contribuyeron en el análisis los tipos de lectura exploratoria y secuencial a través de las cuales se estudiaron los textos para la contextualización teórica de carácter interpretativo y los elementos de la corrección de estilo. En este proceso resultó fundamental la información recogida del corpus lingüístico -breves fragmentos de textosde donde se obtuvieron los errores frecuentes.

La unidad de análisis central fue la tesis de la escritura y los elementos presentes en la corrección de estilo, de allí que se contextualizó con el nivel ontológico de Echeverría (2010). Igualmente, fueron consideradas las características y tipo oracional, según la UNESCO (1983). Por su importante vinculación temática se asumieron además como postura ontoepistémica los aportes de Gadamer (2006), King, (2001) y Ricoeur (2003).

En cuanto al diseño de la investigación, el propósito estuvo centrado en estudiar el contenido de las teorías, además del corpus lingüístico con miras a la interpretación de los fenómenos emergentes. Otra acción metodológica se dedicó a la organización de los artículos a los que se le aplicó el criterio de selección por su relevancia con el tema en estudio y actualización del contenido. La triangulación teórica y el resultado del análisis dieron lugar al momento conclusivo al que hemos llamado componentes intrínsecos al proceso de corrección de estilo.

\section{Proposiciones ontológicas}

La interpretación se fundamenta en lo ontológico y para ello se asume lo señalado por Echeverría, E. (2010), quien define la ontología desde el "dasein" como el modo particular del ser como somos los seres humanos. Por su pertinencia con este escrito, asumimos el primer postulado básico de la ontología del lenguaje en el que se conceptualiza que interpretamos a los seres humanos como seres lingüísticos que viven en el lenguaje y es justamente ese lenguaje el que resulta clave para comprender los fenómenos humanos. El segundo postulado, igualmente de carácter ontológico, enfatiza que el lenguaje no solo nos permite hablar de las cosas, sino que hace que las cosas sucedan y de allí que el ya referido Echeverría insista en llamarlo generativo porque además de permitir hacer una descripción de la realidad, también la crea. En el marco de enfatizar que el lenguaje es acción volvemos a la premisa de que el lenguaje crea realidades. Por su parte, el tercer postulado señala que los seres humanos se crean a sí mismos en el lenguaje y a través de él. Este postulado resulta muy interesante al distinguir que el interés de la lingüística y la filosofía del lenguaje se enfocan en el mismo lenguaje a diferencia de la ontología que, centra su foco de atención en los seres humanos (Echeverría, 2010).

En torno al estudio y evolución sobre la universalidad del lenguaje se entiende que la lengua y el pensamiento guardan relación con el mundo real, de allí su constante búsqueda por comprender la naturaleza y el pensamiento humano. Sustenta esta afirmación lo expuesto por el lingüista estadounidense Jackendoff (2002), al presentar 
tres apartados; el primero se lo dedica a la teoría de la competencia como una caracterización funcional de las estructuras de datos almacenadas y ensambladas en la mente durante el curso del lenguaje. El ya referido autor, en un segundo apartado, atribuye a la teoría de desempeño una caracterización funcional del uso de estas estructuras de datos mientras ocurre la percepción y producción del lenguaje; un tercer lugar es otorgado a la teoría de la instanciación neuronal que anuncia cómo las estructuras de datos son ensambladas y realizadas en el cerebro.

La interpretación de los seres humanos como seres lingüísticos nos conduce al planteamiento relacionado con el principio de conexión a través del cual el escribiente podrá armar el rompecabezas del tejido escritural consciente de que el lenguaje es un sistema de signos y que por medio de éste podrá significar el conocimiento y la abstracción del saber. Ahora bien, en el contexto de escribir un artículo se entiende que ya hemos cubierto de la investigación: la aplicación de instrumentos, tenemos los hallazgos, la discusión y el cierre. Es así, entonces, que estamos en la fase de divulgación.

\section{Características y tipo oracional}

Para lograr lo señalado en el párrafo anterior han sido considerados los aportes de la Unesco (1983), especialmente cuando define como publicación de carácter primario aquella forma textual análoga con el artículo de investigación, artículo científico y artículo de reflexión. En cuanto a las características de estilo en los artículos científicos refiere la claridad en relación con la sencillez y su pretensión se basa en procurar una rápida comprensión. La precisión es llevada al plano de exactitud para evitar la ambigüedad. En este mismo sentido, hay una mención importante que apunta a la sobriedad inclinada hacia la moderación en clara oposición a la verbosidad o exceso de palabras en una oración. Por su parte, la fluidez resulta en un orden lógico contrapuesto a la fragmentación ya que pretende la cohesión entre las ideas. Distingue también en este contexto, la efectividad, relacionada con la persuasión orientada a alcanzar sus objetivos. Siguiendo con la Unesco (1983), se destaca el tipo de oración, un ejemplo (Ej.) y la sugerencia (s) de aplicación en el marco del orden de un discurso escrito.

- Oración declarativa afirmativa. Ej: la corrección de estilo garantiza la escritura exitosa en la divulgación de un artículo científico. S: Definitivamente este tipo de oración debe predominar a lo largo del escrito. Dado que estamos en el escenario de divulgar un hallazgo o el producto de una consulta documental, se entiende que el mismo lo convierte en una contribución al conocimiento científico. En cualquier caso, escribiremos de forma afirmativa.

- Oración declarativa negativa. Ej: el diseño de la corrección de estilo no hace sino corroborar la importancia de la revisión. S: Por el nivel de ambigüedad y carencia de consistencia entre las ideas expuestas, se sugiere no utilizarlas en el escrito.

- Oración interrogativa. Ej: ¿La mayoría de quienes escriben un artículo conocen el diseño de la corrección de estilo? S: Una pregunta puede resultar interesante en 
medio del escrito, pero la sugerencia es que seamos discretos en su uso para evitar excesos que alejen el orden del discurso de la elocuencia o fluidez jerárquica de las ideas.

- Oraciones exclamativas. Ej: se reconoce la importancia de la corrección de estilo. ¡Vaya que es importante! S: Dado su carácter expresivo su presencia resulta tácita; más aún porque es perfectamente detectable por el requisito de incluir los signos de admiración. Utilizar los signos de forma adecuada nos evita dar explicación más allá de lo que deseamos expresar.

- Oraciones dubitativas. Ej: quizás conocer el diseño de corrección de estilo determinaría, para el lector, la importancia de saber aplicarlo. S: Esta modalidad refleja aquello que pueda o no realizarse, que pueda o no ocurrir. Funge de apoyo lo expresado por González (2001), cuando precisa que este tipo de aseveración de carácter dubitativo representa juicios de posibilidad o probabilidad, incluso plantea que es un acto de habla aseverativo atenuado.

- Oraciones exhortativas y desiderativas. Ej: se hace un llamado a la comunidad para que conozca los elementos básicos de la corrección de estilo. Veamos una segunda oración a modo de ejemplo: se espera que los tres apartados de este artículo representen un aporte para cada lector. S: En concreto, usualmente este tipo de oraciones presenta un fundamento para persuadir. Especialmente, cuando se utiliza como estrategia comunicativa guiando al lector hacia la apropiación de una idea nuclear en torno a la temática en estudio.

- Oraciones imperativas. Ej: usa el diseño de la corrección de estilo para optimmizar tu propia escritura. S: En una comunicación para divulgar unos resultados es evidente que el discurso no puede ser un mandato, por el contrario, estamos frente a una comunicación compuesta por espacios dedicados a una fundamentación teórica, metodológica, de discusión y hallazgos.

El proceso de escritura en el contexto de un artículo científico requiere el orden del discurso que permita, de forma expedita, el entendimiento de las ideas. De allí que, la vinculación de las categorías oracionales con el tema del estilo tiene la intencionalidad de ejemplificar, dentro del mismo texto, aquello que por descuido o desconocimiento dejamos de lado cuando divulgamos el resultado de una investigación. Una pregunta de Gadamer (2006) es oportuna en este contexto ¿Hay un lenguaje propio de la ciencia que sea preciso escuchar? Por una parte, la ciencia fija sus propios medios lingüísticos para el entendimiento comunicativo en el proceso de investigación. Por otra parte, la ciencia utiliza un lenguaje que pretende llegar a la conciencia pública.

\section{Enfoque interpretativo}

En aras del rigor epistemológico que se merece el proceso de escritura, resulta imprescindible el reconocimiento de una literatura que aborda el aspecto conceptual en estudio, como señala Mari Mutt (2013). "El artículo científico es un informe escrito que comunica por primera vez los resultados de una investigación. Los artículos científicos publicados en revistas científicas componen la literatura primaria de la ciencia". 
El planteamiento anterior sirve de sustento para vincularlo con los elementos comunicativos presentes en el orden del discurso, entre los que destaca la organización jerárquica para presentar los datos relacionando las ideas, oraciones principales y secundarias, párrafos y texto completo. Se aborda esto, en el contexto de una sociedad digital en la que se evidencia la presencia del hipertexto y del que sabemos que la información puede resultar en una escritura discontinua o expresamente sucesiva. En definitiva, se trata de una escritura que expresa un resultado producto de la utilización de medios comunicativos, según la valoración que hagamos de nuestro entendimiento lingüístico.

El escritor de un artículo científico se propone divulgar un contenido para ser comprendido y, en ese sentido tiene conocimiento explícito del significado de comunicar por medio de un sistema de signos. De acuerdo con esta realidad, el escritor debe analizar la forma cómo anuncia sus ideas. Según su propósito, tiene cuidado en combinar las palabras y presta atención a la construcción de la oración con núcleo y sus respectivos sustentos. Posee la conciencia lingüística de que la ortografía, desde su nivel micro, tiene que ser perfecta. Tener un error ortográfico se convierte para el árbitro en un criterio de rechazo. Podrían estar pensando ¿Qué pasa si se trata de un error tipográfico? En este caso, la responsabilidad igual recae en su autor dada la omisión en el proceso de revisión. Previo a la presentación pública le acarreará serios problemas de credibilidad con el comité evaluador.

King (2001), por su parte, presenta el culmen en torno a este privilegiado tema con dos notables sentencias "Escribir es humano y corregir divino" "El corrector siempre tiene razón". Desde el enfoque atribuido al factor humano podríamos entender que es posible escribir y equivocarnos, es entendible admitir que cometemos errores; pero allí es donde debemos tener presente la importancia de revisar nuestros propios escritos o pedir ayuda a los amigos que sabrán valorar el tiempo y el esfuerzo implicado en el proceso de escribir.

Es perfectamente atribuible el diseño de la corrección de estilo a lo señalado por King (2001) al darle la razón al corrector. En la revisión de la literatura sobre los aspectos conceptuales y característicos del estilo hay opiniones divididas y que incluso afirman categóricamente que un corrector de estilo "modifica" la intención del autor. Estoy totalmente en desacuerdo con esa afirmación, el diseño de corrección de estilo se inserta en lo formal y no en el contenido mismo del documento. En ese sentido, no puede ni debe darse una intervención de quien corrige. Ahora bien, tener en cuenta por ejemplo la revisión sintáctica de una oración, es decir, el orden específico de una oración puede corresponderse con un estilo particular de quien escribe, claro está, sin alterar el orden significativo de lo que necesitamos expresar por medio de la escritura.

El trabajo de un corrector de estilo implica la sutileza de resguardar el significado completo del texto e intención de su autor. Éste se pasea por los distintos espacios y recovecos del escrito. Ese recorrido conlleva vestirse como el propio investigador al 
tiempo de reconocer aquello oculto culpable de oscurecer la integridad del lenguaje. Significa que el propio autor no detecta la telaraña que resulta de una palabra ausente 0 repetida. Puede ser que no advierta la inexistencia de un conectivo en su función cohesionadora impidiendo la articulación gramatical y léxica.

En un sentido interpretativo, el corrector asume lo señalado por Ricoeur (2003), "Toda comprensión óntica u ontológica se expresa, ante todo y desde siempre en el lenguaje. Por lo tanto, no es en vano buscar del lado de la semántica un eje de referencia para todo el conjunto del campo hermenéutico". De acuerdo con esta sentencia, resulta esencial concebir la escritura desde una posición epistémica del lenguaje como medio de expresión a través del cual se distribuye un tejido de ideas a favor de un artículo científico.

De manera consecuente con el análisis interpretativo, seguimos con el ya referido Ricoeur (2019), quien señala "Hay un modo de ser que existe al comprender y sobreviene justo después de la interrogación al ser que es el "ahí" de todo ser, es el Dasein". Desde una reflexión lingüística aplicada al aspecto de forma en la escritura, consideramos pertinente la contribución ricourniana cuando señala que la comprensión de expresiones multívocas o simbólicas es un momento de la comprensión del sí. Por su parte, el sujeto que se interpreta al interpretar los signos es un existente que se descubre puesto en el ser antes de que se sitúe y posea.

En este sentido, sirva el párrafo anterior para señalar que el proceso interpretativo se constituye en buscar la raíz ontológica de la comprensión como resultado del acontecimiento que ocurre en el lenguaje y es a través de éste que se alcanza el medio de una realidad universal que da lugar al todo ser de la comprensión. Este planteamiento se impone a la necesidad de apropiación suscitada al conocer los componentes intrínsecos de la escritura coadyuvantes en el proceso de la corrección de estilo a partir de su uso en la producción de un artículo científico.

\section{Momento conclusivo. Errores frecuentes}

La corrección de estilo implicó una lectura analítica de las ideas expuestas en medio del orden discursivo de cada artículo. De esta manera se ha interpretado la valoración de la especificidad de acceso lingüístico dispuesto en los componentes intrínsecos al proceso de corrección de estilo, como podemos apreciar en las siguientes líneas.

De forma exagerada en el orden ortográfico se detectaron el uso inadecuado de mayúscula sostenida y palabras sin tilde. Entre los vicios del discurso se encontró el pleonasmo, por ejemplo, se indagó para investigar. Un método jamás visto antes. También se ha identificado el uso extralimitado de pronombres relativos como: los cuales, las cuales. Otro error frecuente se refiere al uso reiterado del gerundio como inicio de párrafo y la inserción del mismo conectivo a lo largo de todo el trabajo.

292 Enfoque interpretativo en la corrección de artículos científicos - Eduweb, 2021, septiembre-diciembre, v.15, n.3. /286-294 
En el análisis del corpus lingüístico se advirtió la confusión gramatical que se generó en el escribiente cuando usó el verbo "haber" previo al participio pasado, cuyas terminaciones son "ado" "ido"; específicamente omitieron o sustituyeron la letra "h" por la preposición "a". Fueron errores: "Se a visto". "Se a iniciado" en lugar de: Se ha visto. Se ha iniciado.

Resultó frecuente tropezar con expresiones que buscaban recalcar el significado de una palabra, pero en realidad lo que ocasionó fue la alteración de la calidad semántica de la oración. Algunos ejemplos: más importante; pandemia mundial; buscó indagar; las que tuvieron más realce; verdaderamente grande; repetidas veces; surgidos hallazgos; tan importante. De todo este planteamiento se dice que esto generó. Para este último caso se sugiere: A partir de este planteamiento. Ahora bien, fijemos la atención en las palabras con formato cursivo; sin duda son prescindibles para optimizar el significado de la expresión lingüística.

En torno al análisis, se encontró también un fenómeno lingüístico que se relaciona con el hipérbaton, que es definido como una figura retórica empleada en la poesía para alterar el orden lógico de un verso. Este mismo recurso, de carácter sintáctico, distorsionó la disposición de las palabras en medio de las oraciones. Por ejemplo: "Se justifica esta investigación". "Lo expresado en concordancia". En lugar de: Esta investigación se justifica. En concordancia con lo expresado.

\section{Otros errores comunes encontrados en el corpus seleccionado}

$\checkmark$ "La empresa pequeña, mediana y grande empresas..." La empresa pequeña, mediana y grande.

$\checkmark$ "Recogió informaciones". Recogió información.

$\checkmark$ "Ya han transcurrido más de un año". Ya ha transcurrido un año.

$\checkmark$ "En base a informaciones recientes". Con base en las informaciones recientes.

$\checkmark$ "Se toma el enfoque interpretativo". Se asume el enfoque interpretativo

$\checkmark$ "Datos evidenciales". Datos evidenciables.

$\checkmark$ "Pérez y Arrieta expresa que". Pérez y Arrieta expresan que.

$\checkmark$ "Lo señalado por los autores reflejan". Lo señalado por los autores refleja.

$\checkmark$ "Refleja la información señalada". Refleja la información.

$\checkmark$ "El $20 \%$ señalaron". El $20 \%$ señaló.

$\checkmark$ "Los estudiantes de cuarto grado, los cuales". Los estudiantes de cuarto grado quienes...

$\checkmark$ "Sobre todo". Especialmente

$\checkmark$ "Los resultados muestran muchas inconsistencias". Los resultados muestran inconsistencias.

$\checkmark$ "Las cuales se realizarán" Que se realizarán

$\checkmark$ "Los cuales se necesitan". Que se necesitan

$\checkmark$ "La palabra más relacionada con". La palabra relacionada con.

$\checkmark$ "Ese resultado tiene muchas connotaciones". Ese resultado tiene connotaciones. 
$\checkmark$ "Las ventajas más resaltantes". Las ventajas.

En conclusión, la intención de este breve análisis ha sido ofrecer una ruta de lectura para la valoración de la especificidad lingüística dispuesta en los componentes intrínsecos al proceso de corrección de estilo. El corrector busca en las profundidades del lenguaje toda posibilidad de comprensión íntegra con el firme propósito de decantar el enjambre de oraciones cuyas palabras aparezcan desordenadas o duplicadas; así como expresiones típicas de la oralidad, errores ortográficos, la transposición de letras y todo fenómeno emergente en consideración de la dinámica del lenguaje.

\section{Referencias}

Echeverría, E. (2010). Ontología del lenguaje. Buenos Aires: Granica editor.

Berelson, B. (1967). Contex analisys en Lindzey: Handbook of social psyshology. Tomo I. New York, Lindzey.

Gadamer, H. (2006). Verdad y método. Tomo II. Ediciones Sígueme: Salamanca.

González, C. (2001). Revisión de la clasificación de la oración según el "modus". Universidad de Extremadura. Anuario de estudios filológicos. Fundación Dialnet. En [https://dialnet.unirioja.es/servlet/autor?codigo=85580]

Jackendoff, R. (2002). Foundations of language. Brain, meaning. Grammar, evolution. Oxford University Press Inc., New York.

King, S. (2001). Mientras escribo. Ramdom House: Barcelona.

Mari Mut, J.A. (2013). Redacción científica. Ediciones digitales info. En [http://edicionesdigitales.info/Manual/manual.pdf]

Ricoeur, P. (2003). El conflicto de las interpretaciones. Ensayos de hermenéutica. Fondo de cultura econóimca: Argentina.

UNESCO. (1983). Guía para la redacción de artículos científicos destinados a la publicación (2 ed.). París. En: [https://es.slideshare.net/CarlosGonzalez256/guiaunesco] 